\title{
Risk of cataract development among children with juvenile idiopathic arthritis-related uveitis treated with topical corticosteroids
}

\author{
Jennifer E. Thorne, MD, PhD ${ }^{1,2}$, Fasika A. Woreta, MD, MPH ${ }^{1}$, James P. Dunn, $\mathbf{M D}^{1}$, and \\ Douglas A. Jabs, MD, MBA 2,3 \\ ${ }^{1}$ Department of Ophthalmology the Johns Hopkins University School of Medicine, Baltimore, MD \\ ${ }^{2}$ Department of Epidemiology the Johns Hopkins University Bloomberg School of Public Health, \\ Baltimore, MD \\ ${ }^{3}$ Departments of Ophthalmology and Medicine, the Mount Sinai School of Medicine, New York, NY
}

\begin{abstract}
Purpose-To investigate the risk of cataract development among patients with juvenile idiopathic arthritis (JIA)-associated uveitis treated with topical corticosteroids.
\end{abstract}

Design-Retrospective cohort study

Participants-75 patients with JIA- associated uveitis observed from July 1984 through August 2005 at a single academic center.

Methods-Clinical data on these patients were collected by chart review and were analyzed.

Main outcome measures-Incidence of new-onset cataract. Risk factors for cataract development were assessed with attention paid to the use of topical corticosteroids.

\begin{abstract}
Results-Over a median follow-up of 4 years, the incidence of new-onset cataract was 0.04/eyeyear (EY, 95\% confidence interval [CI]: 0.02/EY, 0.09/EY). Of the 60 eyes in 40 patients who received topical corticosteroid therapy, there was a dose-dependent increase in the rate of cataract development among eyes receiving topical corticosteroids. The incidence of cataract was $0.01 / \mathrm{EY}$ for eyes treated with $\leq 3$ drops daily and $0.16 / \mathrm{EY}(\mathrm{P}=0.0006$ for $\log$ rank test) for eyes treated with $>3$ drops daily. Among eyes receiving $\leq 2$ drops daily, the incidence of cataract was 0/EY (95\% CI, one-sided: $0.03 / \mathrm{EY}$ ). Presence of posterior synechiae, active uveitis, and use of topical corticosteroids at presentation were statistically significantly associated with cataract development after controlling for confounding variables. Use of topical corticosteroids was associated with cataract formation independent of uveitis activity. Using longitudinal data analysis and controlling for duration of uveitis, presence and degree of active uveitis, and concomitant use of other forms of corticosteroids in a time-updated fashion, treatment with $\leq 3$ drops daily of topical corticosteroid was associated with an $87 \%$ lower risk of cataract formation compared to eyes treated with $>3$ drops daily (relative risk $=0.13,95 \%$ CI: $0.02,0.69, \mathrm{P}=0.02$ ).
\end{abstract}

Corresponding Author: Jennifer E. Thorne, MD, PhD; Department of Ophthalmology, The Wilmer Eye Institute; 550 North Broadway; Suite 700; Baltimore, MD 21205, Phone: (410) 955-1966; Fax: (410) 955-0629; jthorne@ jhmi.edu.

Publisher's Disclaimer: This is a PDF file of an unedited manuscript that has been accepted for publication. As a service to our customers we are providing this early version of the manuscript. The manuscript will undergo copyediting, typesetting, and review of the resulting proof before it is published in its final citable form. Please note that during the production process errors may be discovered which could affect the content, and all legal disclaimers that apply to the journal pertain. 
Conclusions - In our cohort, topical corticosteroid use was associated with an increased risk of cataract formation independent of active uveitis or presence of posterior synechiae. However, chronic use of topical corticosteroids dosed at $\leq 3$ drops daily appeared to be associated with a lower risk of cataract development relative to eyes receiving higher doses over follow up in the setting of suppressed uveitis.

\section{Introduction}

Juvenile idiopathic arthritis (JIA) comprises a group of arthritides of unknown cause with an onset before 16 years and persisting for 6 weeks or longer. ${ }^{1,2}$ The uveitis of JIA in these patients typically is a chronic, bilateral, nongranulomatous, and asymptomatic anterior uveitis. The frequency of severe visual impairment has been reported in $18 \%$ to $38 \%$ of patients, ${ }^{3-8}$ and the rate of 20/200 or worse visual acuity in eyes with JIA-related uveitis has been reported as $0.08 /$ eye-year (EY) or 0.09/person-year (PY). ${ }^{9}$

Cataract is a frequent complication of JIA-related uveitis, ${ }^{1,6-8}$ and in one study, ${ }^{9}$ it accounted for almost two-thirds of incident blindness (20/200 or worse vision) among affected eyes.

Cataract may result either from uncontrolled uveitis or from the use of corticosteroids, the risk of which is increased by local corticosteroids such as drops or periocular injections. ${ }^{5,6,9,10}$

Because children with JIA-related uveitis often receive topical corticosteroids over extended periods of time to suppress their uveitis, it would be important to have estimates of the risk of cataract from topical corticosteroid therapy and to estimate whether there is a dose response or a threshold dose that modifies the risk of cataract development.

In order to address these questions, we analyzed the incidence of and risk factors for cataract development, and the effect of treatment with topical corticosteroids on the development of cataract in a cohort of 75 consecutive patients with JIA-associated chronic uveitis.

\section{Methods}

\section{Study population}

All patients with JIA-associated uveitis who were seen at the Division of Ocular Immunology at the Wilmer Eye Institute, Johns Hopkins School of Medicine between July 1984 and August 2005 were included in the cohort. Details of the study population have been described previously. 8,9 The study was performed with the approval of the Johns Hopkins University School of Medical Institutional Review Board.

\section{Data collection}

Information on all patients evaluated for JIA-related chronic uveitis was collected. Data included demographics, ophthalmologic examination, and medications received at each clinic visit. Details of the ophthalmologic examination have been described previously but it should be noted that the grade of uveitis activity (ranging from no cells to 4+ cells in the anterior chamber) was recorded for each eye at each clinical visit. ${ }^{8,9}$ Use of corticosteroids (including the dose and route of administration) and of immunosuppressive drugs were collected at each visit including the number of topical corticosteroid drops taken daily at the time of the visit. The therapy for each patient was managed according to best medical judgment using guidelines that have been subsequently published. ${ }^{11,12}$ Data collection was facilitated by the use of flow sheets to ensure complete clinical data at every visit and a divisionally standardized approach to corticosteroid tapering, use of immunosuppressive drugs, and follow up was utilized. 8,9 , 13-15 Data were entered on a computer-based standardized database for statistical analysis. 


\section{Main outcome measures}

The incidence of cataract development was assessed. Cataract was defined as the presence of $1+$ nuclear sclerosis or 1+ cortical change or trace posterior subcapsular changes. Although our definition is arbitrary, it is consistent with the definition that we have used in previous papers that report clinical outcomes in this cohort of patients with JIA-related uveitis. ${ }^{8}{ }^{8}$ Risk factors for the development of cataract also were assessed with attention paid to the use and daily dosage of topical corticosteroids.

\section{Statistical analysis}

Incidence of cataract was calculated as the number of events divided by the number of patients or eyes at risk. Incidence rates for different treatment regimens (e.g., different daily doses of topical corticosteroids) were compared using time-updated survival analyses. ${ }^{16}$ For these analyses, treatment regimens (the exposure variable) and the development of cataract (the outcome variable) were updated and recorded for each clinical visit for affected eyes of each patient. In this way, a patient's eye(s) with uveitis might contribute eye-time to various treatment groups. Multivariate, time-dependant Cox regression models were used to assess the risk of developing new-onset cataract among patients with JIA-associated uveitis receiving variable doses of topical corticosteroids. ${ }^{17}$ The number of drops of corticosteroids taken daily was updated at each clinical visit and the models controlled for presence and degree of active inflammation, duration of uveitis, number of uveitis relapses, and concomitant oral corticosteroid and/or immunosuppressive drug use. Secondary models including baseline variables that were statistically significantly associated with incident cataract development in the univariate analyses also were constructed with particular interest in the effect of the presence of posterior synechiae. Goodness of fit tests were employed and the most parsimonious model was presented. Analyses were performed as "by eye" analyses in which all affected eyes at risk were included in the analyses. All Cox regression models used robust variance estimation to account for correlation between eyes in patients with bilateral uveitis.

${ }^{18} \mathrm{P}$-values were 2-sided and nominal. Analyses were performed using Intercooled Stata 9.0 statistical software (Stata Corporation, College Station, TX).

\section{Results}

\section{Study Population}

Characteristics of the study population at presentation are summarized as Table 1 and have been reported previously. 8,9 Overall, the prevalences of visual impairment and blindness at presentation were $36.4 \%$ and $23.7 \%$ of eyes, respectively. Two-thirds of eyes had at least one structural ocular complication. A cataract was present in $22.5 \%$ of eyes at the presenting clinical examination. An additional $23.1 \%$ of eyes already had undergone cataract surgery by the time of the presenting clinical examination. Approximately $45 \%$ of eyes had active uveitis $(\geq 0.5+$ cells or greater in the anterior chamber) at the time of presentation. Over $85 \%$ of patients $(90 \%$ of eyes) were treated with prednisolone acetate $1 \%$. The rest were largely treated with rimexolone $1 \%$. Nine patients were receiving oral corticosteroid therapy at presentation. An additional 10 patients were exposed to oral corticosteroids during the observed follow up period; however the overall duration of follow up time for which patients were exposed to oral corticosteroids was low (17\% of the total person-time observed) and the daily dose of oral corticosteroids also was low (median $=8 \mathrm{mg}$ daily). Despite the fact that our patients were exposed to low daily doses of oral corticosteroids for relatively short durations of time, we controlled for use of oral corticosteroids in each of the analyses investigating the potential association between topical corticosteroid therapy and cataract formation. 


\section{Risk factors for the development of cataract}

The median follow up period was 4 years (mean follow up: 4.6 years, range: 0.5 to 15 years). The overall incidence of newly diagnosed cataract was $0.04 /$ eye-year (EY, 95\% confidence interval [CI]: 0.02/EY, 0.09/EY) and 0.07/person-year (PY, 95\% CI: 0.02/PY, 0.14/PY). Risk factors for cataract development are summarized as Table 2 . After controlling for potentially confounding variables, the presence of posterior synechiae, active uveitis $(\geq 0.5+$ cells in the anterior chamber), and use of topical corticosteroids at the time of presentation to our clinic all were statistically significant risk factors for the development of new-onset cataract during the follow up period. Increasing severity of anterior chamber inflammation was associated with an increasingly greater risk of cataract development in the multivariate analysis (P-value for trend $=0.005$ in the multivariate analysis). Furthermore, a second multivariate analysis that evaluated any active uveitis at baseline ( $\geq 0.5+$ cells in the anterior chamber) demonstrated a statistically significant increased risk in cataract development (relative risk [RR] in the multivariate analysis $=6.04,95 \% \mathrm{CI}: 1.24,29.5, \mathrm{P}=0.03$ ). Female gender was associated with a decreased risk of cataract development in the univariate analysis but not in the multivariate analysis $(\mathrm{RR}$ in the multivariate analysis $=1.02, \mathrm{P}=0.65)$. We were not able to assess the effect of a history of intraocular surgery prior to presentation to our clinic on the development of cataract as there were no eyes with this exposure variable that were at risk for developing cataract (i.e., all eyes of all patients with a history of intraocular surgery prior to presentation either already had developed cataracts or had undergone cataract surgery prior to presentation to our clinic).

\section{Effect of topical corticosteroids on the development of cataract among children with JIA- associated uveitis}

In order to evaluate the degree to which the daily dose of topical corticosteroids affected the development of cataract, we calculated the incidence of new-onset cataract among eyes of patients treated with various daily doses of topical corticosteroids. Because the dosing of topical corticosteroids may change over time, the dose of topical corticosteroids was collected at each clinical visit and time-updated survival analyses employed to calculate the incidence of cataract for each dose. Sixty eyes of 40 patients had available follow up and were treated with topical corticosteroids. The incidence of cataract among eyes of patients receiving zero, one, or two drops daily of topical corticosteroids was zero; the upper bound of the $95 \%$ confidence interval for the incidence of cataract at $\leq 2$ drops daily topical corticosteroids was $0.03 / E Y$. For eyes treated with topical corticosteroids three times daily, the rate of cataract development was 0.01 / EY (95\% CI: 0.005/EY, 0.03/EY). The rate of cataract development for eyes receiving topical corticosteroids four times daily was $0.07 / \mathrm{EY}$ ( $95 \%$ CI: 0.02/EY, 0.14/EY). For eyes receiving doses of greater than four times daily (range: 5-12 times daily), the rates of cataract development were similar; thus the groups were combined yielding a cataract incidence rate of 0.16/EY (95\% CI: 0.09/EY, 0.21/EY). In order to control for the effect of other forms of corticosteroids, use of immunosuppressive drug therapy, and the presence of and degree of active uveitis on the development of cataract, multivariate regression models utilizing timeupdated data were constructed. Eyes treated with topical corticosteroids administered at doses of $\leq 3$ drops daily had an $87 \%$ reduced risk for the development of cataract $(R R=0.13,95 \%$ CI: $0.02,0.69, \mathrm{P}=0.02)$. Additional multivariate analyses were employed that controlled for the varying degree of anterior chamber inflammation, number of uveitis recurrences, for duration of uveitic disease, and for characteristics at presentation that were statistically significantly associated with cataract development (see Table 2); and these analyses yielded a similar decreased risk for cataract development among eyes treated with topical corticosteroid doses of $\leq 3$ drops daily. The most parsimonious multivariate model is presented as Table 3 .

Because we were unable to assess the progression of cataract, we performed a subgroup analysis to assess the risk of cataract surgery among eyes treated with topical corticosteroids 
as a surrogate marker for cataract progression. The risk of cataract surgery was $68 \%$ lower for eyes receiving $\leq 3$ drops daily of topical corticosteroids ( $R R=0.32 ; P=0.05)$; however, the results were not statistically significant after controlling for potential confounding $(R R=0.37$; $\mathrm{P}=0.28)$, presumably related in part to the small number of events $(\mathrm{N}=14$ eyes undergoing cataract surgery during the observed follow up period).

\section{Discussion}

JIA-associated uveitis frequently has been reported to result in visual impairment as a result of structural ocular complications such as cataract. ${ }^{1,6,7,9,19}$ We reviewed our experience with JIA-associated uveitis over 21 years in order to estimate in the effect of treatment with topical corticosteroids on the development of cataract over time. Because of the retrospective design of this study, the results must be interpreted with caution. A referral bias may exist because our institution is a tertiary care medical center, and it is likely that more severe cases of JIAassociated uveitis were referred to our center as suggested by high frequencies of ocular complications and poor visual acuity, and by the long duration of uveitis prior to presentation to our clinic. ${ }^{8}$ However, the frequency of cataract and other ocular complications at presentation in our cohort ${ }^{8}$ was similar to that reported from other tertiary care centers, ${ }^{5,6}$ but was $\sim 33 \%$ higher than that found in a population-based study in which the frequency of ocular complications at presentation was $45 \% .{ }^{20}$ In some analyses such as those evaluating each grade of anterior chamber inflammation, the number of events was small, which limited the precision of relative risks for these characteristics and may have increased the likelihood of a type II error (i.e., the inability to detect a statistically significant difference when one in fact exists). Due to its retrospective nature, our study lacked a protocol for grading the progression of cataract or for treating active uveitis. However, our clinic employees a divisionally standardized approach to the treatment of uveitis including a standardized tapering schedule for topical corticosteroids and follow up schedule. It is also possible that patients with more severe disease or those exposed to higher doses of topical corticosteroids, oral corticosteroids, or immunosuppressive drug therapy may have been seen more frequently than patients with milder disease and thus, the likelihood of ascertaining the outcome of interest (e.g., new-onset cataract) could have been greater for these patients. However the rate of follow up visits for patients receiving only topical corticosteroids was similar to the rate of visits for those patients receiving oral corticosteroids or immunosuppressive drugs, suggesting that any bias in ascertainment of cataract development was limited. The above potential limitations aside, our study suggests that the risk of cataract development is lower among eyes treated with a dose of topical corticosteroids $\leq 3$ drops daily as compared to higher daily doses of topical corticosteroids after controlling for active uveitis, use of other forms of corticosteroids, and use of immunosuppressive drug therapy.

The incidence rate of new-onset cataract in our cohort was $0.04 / \mathrm{EY}$, which is approximately one-half the rate of cataract development in adults with posterior and panuveitides observed in our clinic (0.08/EY to $0.10 / Y$ depending on series). ${ }^{13-15}$ After controlling for potential confounding, active anterior chamber inflammation at increasing levels and the presence of posterior synechiae at presentation were statistically significantly associated with the development of cataract (Table 2). The presence of posterior synechiae at presentation has been reported previously to be associated with the development of ocular complications and visual loss, ${ }^{9,21}$ and specifically with cataract formation, presumably because the presence of posterior synechiae is a surrogate marker for more severe uveitic disease. ${ }^{21-23}$ Furthermore, one study has reported that patients with JIA-related uveitis who present to the ophthalmologist with posterior synechiae are less likely to undergo remission of the uveitis and therefore typically require long-term therapy with topical corticosteroids even if the uveitis is mild. ${ }^{21}$ In our cohort the presence of posterior synechiae and active inflammation were stronger risk factors for developing cataract over time than was the use of corticosteroids; thus supporting the premise 
that suppression of the uveitis is critical to avoiding the development of ocular complications, such as cataract, among these patients. 8,9

Increasing duration of uveitis at the presenting examination was associated with a decreased risk of cataract development during follow up after controlling for confounding variables. Although this result initially appears to be counter-intuitive, it likely represents a form of survivor bias whereby eyes with milder disease do not develop cataract as rapidly and therefore were without cataract at presentation. The patients with more severe disease likely already had cataract or cataract surgery at the time of presentation and thus, were censored for the analyses of cataract development during follow up. Furthermore, any effect of eyes with longer duration of uveitis or more severe disease who had not developed cataract by the time they presented to our clinic were controlled for at least in part by including the grade of anterior chamber inflammation and the presence of posterior synechiae at presentation in the multivariate analysis.

Longitudinal data analysis was utilized to assess the effect of variable daily doses of topical corticosteroids on the development of cataract during follow up in an effort to better determine a daily dose associated with a reasonably low risk of cataract development if topical corticosteroids are required chronically. We were able to control with each flare of uveitis (including the severity of the anterior chamber inflammation) and for the concomitant use of other forms of corticosteroids, both known to cause cataract, ${ }^{24}$ and for use of immunosuppressive drug therapy which has been reported to slow cataract development and progression. ${ }^{23}$ After controlling for these confounding variables, use of topical corticosteroids $\leq 3$ drops daily was associated with an $87 \%$ reduction in the risk of new-onset cataract when compared to 4 drops daily or more. Although the risk of cataract development among eyes of patients receiving topical corticosteroids $\leq 3$ drops daily was not zero (rate $=0.01 / \mathrm{EY}$ ), our data imply that it may be a reasonable threshold, as the risk of cataract development was substantially higher with higher doses of topical corticosteroids.

Our follow up was moderately long (median of 4 years, range 6 months to 15 years), but we cannot assess the effect of decades of topical corticosteroid therapy. It is possible that the cumulative dose of topical corticosteroid could affect the risk of cataract. However, in our cohort the cumulative dose of topical corticosteroids was higher in eyes that did not develop cataract as compared to eyes that did develop cataract (6810 drops versus 3050 drops; $\mathrm{P}<$ 0.001); therefore a higher cumulative dose among patients taking topical corticosteroids $>3$ drops daily or among those developing cataract could not explain this study's findings.

Analyses involving cumulative doses of topical corticosteroids are subject to biases such as variable dose velocity (e.g., higher daily doses over short periods of time at times of uveitis activity), variable duration of follow up (which was longer among patient with eyes that did not develop cataract in our cohort), and variable ascertainment of daily dosing in between follow up visits (e.g., tapering of topical corticosteroid drops performed by other ophthalmologists participating in the care of the patient), making these analyses less helpful in estimating a threshold dose for clinical use on a chronic basis. Nevertheless it is possible that with very long follow up (e.g., > 10 years) the rate of cataract development could change and even doses of topical corticosteroids $\leq 3$ drops daily might appear less safe.

Furthermore because of a lack of standardization of cataract grading, we were unable to assess progression of cataract among eyes of patients who presented to our clinic with cataract. We performed a subgroup analysis to assess the risk of cataract surgery among eyes treated with topical corticosteroids, which allowed those eyes with cataract at presentation to be included in the analysis. The results were similar to those reported for risk of new-onset cataract with a $68 \%$ reduction in cataract surgery risk for eyes receiving $\leq 3$ drops daily of topical corticosteroids $(\mathrm{RR}=0.32 ; \mathrm{P}=0.05)$; however, the results were not statistically significant 
after controlling for potential confounding. It is likely that because the number of eyes that underwent cataract surgery during follow up was small $(\mathrm{N}=14)$ that the sample size for this analysis was not adequate to achieve conventional statistical significance despite the rather substantial effect size observed. Future prospective studies observing larger populations of patients with JIA-associated uveitis and utilizing standardized cataract grading protocols would be helpful to further investigate the effect of topical corticosteroid therapy on the progression of cataract among these patients.

In summary, presence of posterior synechiae, active uveitis, and use of topical corticosteroids at presentation were statistically significant risk factors for the development of cataract after controlling for other potentially confounding variables. The risk of cataract increased as the number of drops of topical corticosteroids received daily increased in a somewhat dosedependent fashion; this risk increased dramatically between dosages of 3 times daily (rate of cataract $=0.01 / \mathrm{EY}$ and 4 times daily (rate of cataract $=0.07 / \mathrm{EY}$ ) and was independent of uveitis activity, severity, duration, and number of relapses of uveitis. Eyes receiving $\leq 3$ drops of topical corticosteroid daily had an $87 \%$ reduced risk of developing a new cataract during follow up compared to eyes treated with higher daily doses. These data imply that in the setting of suppressed uveitis, patients with JIA-associated chronic anterior uveitis may be treated with low doses of topical corticosteroids ( $\leq 3$ drops daily) over moderate periods of follow up with a low risk of developing cataract.

\section{Acknowledgments}

Funding: Supported by grants EY-13707 (Dr. Thorne) and EY-00405 (Dr. Jabs) from the National Eye Institute, Bethesda, Maryland. Dr. Thorne is the recipient of the Research to Prevent Blindness Sybil Harrington Special Scholars Award. Supported in part by the Kids' Uveitis Research and Education (K.U.R.E.) Fund, Baltimore, Maryland.

\section{References}

1. Kotaniemi K, Savolainen A, Karma A, Aho K. Recent advances in uveitis of juvenile idiopathic arthritis. Surv Ophthalmol 2003;48:489-502. [PubMed: 14499817]

2. Petty RE, Southwood TR, Manners P, et al. International League of Associations for Rheumatology. International League of Associations for Rheumatology classification of juvenile idiopathic arthritis: second revision, Edmonton, 2001. J Rheumatol 2004;31:390-2. [PubMed: 14760812]

3. Key SN III, Kimura SJ. Iridocyclitis associated with juvenile rheumatoid arthritis. Am J Ophthalmol 1975;80:425-9. [PubMed: 1172374]

4. Özdal PC, Vianna RN, Deschênes J. Visual outcomes of juvenile rheumatoid arthritis-associated uveitis in adults. Ocul Immunol Inflamm 2005;13:33-8. [PubMed: 15804767]

5. Dana MR, Merayo-Lloves J, Schaumberg DA, Foster CS. Visual outcomes prognosticators in juvenile rheumatoid arthritis-associated uveitis. Ophthalmology 1997;104:236-44. [PubMed: 9052627]

6. Kump LI, Cervantes Castañeda RA, Androudi SN, et al. Visual outcomes in children with juvenile idiopathic arthritis-associated uveitis. Ophthalmology 2006;113:1874-7. [PubMed: 16884776]

7. Yu EN, Meniconi ME, Tufail F, et al. Outcomes of treatment with immunomodulatory therapy in patients with corticosteroid-resistant juvenile idiopathic arthritis-associated chronic iridocyclitis. Ocul Immunol Inflamm 2005;13:353-60. [PubMed: 16419420]

8. Woreta F, Thorne JE, Jabs DA, et al. Risk factors for ocular complications and poor visual acuity at presentation among patients with uveitis associated with juvenile idiopathic arthritis. Am J Ophthalmol 2007;143:647-55. [PubMed: 17224116]

9. Thorne JE, Woreta F, Kedhar SR, et al. Juvenile idiopathic arthritis-associated uveitis: incidence of ocular complications and visual acuity loss. Am J Ophthalmol 2007;143:840-6. [PubMed: 17362866]

10. Lafranco Dafflon ML, Tran VT, Guex-Crosier Y, Herbort CP. Posterior sub-Tenon's steroid injections for the treatment of posterior ocular inflammation: indications, efficacy and side effects. Graefes Arch Clin Exp Ophthalmol 1999;237:289-95. [PubMed: 10208261] 
11. Jabs DA, Rosenbaum JT, Foster CS, et al. Guidelines for the use of immunosuppressive drugs in patients with ocular inflammatory disorders: recommendations of an expert panel. Am J Ophthalmol 2000;130:492-513. [PubMed: 11024423]

12. Standardization of Uveitis Nomenclature (SUN) Working Group. Standardization of uveitis nomenclature for reporting clinical data: results of the first international workshop. Am J Ophthalmol 2005;140:509-16. [PubMed: 16196117]

13. Thorne JE, Jabs DA, Peters GB, et al. Birdshot retinochoroidopathy: ocular complications and visual impairment. Am J Ophthalmol 2005;140:45-51. [PubMed: 16038650]

14. Bykhovskaya I, Thorne JE, Kempen JH, et al. Vogt-Koyanagi-Harada disease: clinical outcomes. Am J Ophthalmol 2005;140:674-8. [PubMed: 16226518]

15. Thorne JE, Wittenberg SE, Jabs DA, et al. Multifocal choroiditis with panuveitis: incidence of ocular complications and of loss of visual acuity. Ophthalmology 2006;113:2310-6. [PubMed: 16996607]

16. Diggle, P.; Liang, KY.; Zeger, SL. Analysis of Longitudinal Data. Oxford, UK: Clarendon Press; 1994. p. xx-xx.AQ: must provide specific inclusive pagination for material being referenced

17. Cox, DR.; Oakes, D. Analysis of Survival Data. London: Chapman and Hall; 1984. p. 248-75.

18. Lin DY, Wei LJ. The robust inference for the Cox proportional hazards model. J Am Stat Assoc 1989;84:1074-8.

19. Chalom EC, Goldsmith DP, Koehler MA, et al. Prevalence and outcome of uveitis in a regional cohort of patients with juvenile rheumatoid arthritis. J Rheumatol 1997;24:2031-4. [PubMed: 9330949]

20. Heiligenhaus A, Niewerth M, Mingels A, et al. Epidemiology of uveitis in juvenile idiopathic arthritis from a national paediatric rheumatologic and ophthalmologic database [in German]. Klin Monatsbl Augenheilkd 2005;222:993-1001. [PubMed: 16418970]

21. Edelsten C, Lee V, Bentley CR, et al. An evaluation of baseline risk factors predicting severity in juvenile idiopathic arthritis associated uveitis and other chronic anterior uveitis in early childhood. Br J Ophthalmol 2002;86:51-6. [PubMed: 11801504]

22. Wolf MD, Lichter PR, Ragsdale CG. Prognostic factors in the uveitis of juvenile rheumatoid arthritis. Ophthalmology 1987;94:1242-8. [PubMed: 3684202]

23. Sijssens KM, Rothova A, Van de Vijver DA, et al. Risk factors for the development of cataract requiring surgery in uveitis associated with juvenile idiopathic arthritis. Am J Ophthalmol 2007;144:574-9. [PubMed: 17706583]

24. Foster CS, Fong LP, Singh G. Cataract surgery and intraocular lens implantation in patients with uveitis. Ophthalmology 1989;96:281-8. [PubMed: 2629713] 
Table 1

\section{Characteristics of patients with juvenile idiopathic arthritis and uveitis at presentation}

\begin{tabular}{ll}
\hline Patient-specific characteristics & \\
Number of patients & 75 \\
Median age at diagnosis of uveitis, years (range) & $7(1-36)$ \\
Gender, \% girls & 73.3 \\
Race, \% Caucasian & 84.9 \\
Median duration of uveitis prior to presentation, years & 6 \\
Bilateral uveitis, \% & 70.7 \\
JIA type, \% persistent oligoarticular & 81.3 \\
ANA positive, \% & 69.7 \\
Therapies, \% & \\
Topical corticosteroids & 64.0 \\
Oral corticosteroids & 12.0 \\
$\quad$ Methotrexate & 16.0 \\
Cyclosporine & 2.7 \\
Median follow up time, years (mean, range) & $3(4.6,0.5-15)$ \\
Eye-specific characteristics & \\
Number of affected eyes & 132 \\
Any ocular complication ${ }^{*}$ & 67.0 \\
Cataract & 22.5 \\
Aphakic & 17.0 \\
Pseudophakic & 6.1 \\
Anterior chamber cell ( $\geq 0.5+$ cells) & 27.5 \\
Visual acuity, affected eyes, \% & 44.3 \\
20/50 or worse & \\
20/200 or worse & 36.4 \\
\hline & 23.7 \\
\hline
\end{tabular}

ANA $=$ antinuclear antibody; JIA $=$ juvenile idiopathic arthritis.

* Includes band keratopathy, cataract, ocular hypertension, hypotony, optic nerve edema, epiretinal membrane, and macular edema.

Adapted from: Woreta F, et al. ${ }^{8}$ with permission from Elsevier. 
Table 2

Risk factors at baseline associated with cataract development in eyes with juvenile idiopathic arthritis-associated uveitis

\begin{tabular}{|c|c|c|}
\hline \multirow[t]{2}{*}{ Characteristic at presentation } & \multicolumn{2}{|c|}{ Risk of cataract } \\
\hline & Crude RR $\left(95 \%\right.$ CI, P) ${ }^{*}$ & Adjusted RR $(95 \% \text { CI, P })^{\dagger}$ \\
\hline Age at uveitis diagnosis (years) & $1.10(0.96,1.26,0.16)$ & \\
\hline Gender (girls vs boys) & $0.18(0.04,0.80,0.02)$ & \\
\hline Race (nonwhite vs white) & $1.62(0.40,6.57,0.50)$ & \\
\hline Duration of uveitis (years) & $0.85(0.76,0.95,0.005)$ & $0.80(0.70,0.92,0.002)$ \\
\hline Persistent oligoarthritis & $0.96(0.18,5.04,0.96)$ & \\
\hline ANA-positive & $3.57(0.48,26.3,0.21)$ & \\
\hline Posterior synechiae & $25.28(2.88,221.8,0.004)$ & $17.90(3.86,83.2,<0.001)$ \\
\hline \multicolumn{3}{|l|}{ Anterior chamber cell $(\leq \text { rare cells }=\text { reference })^{t}$} \\
\hline $0.5+($ trace $)$ cells & $1.56(0.91,2.68,0.11)$ & $1.73(0.92,3.27,0.09)$ \\
\hline $1+$ cells & $1.75(0.95,3.24,0.07)$ & $1.99(0.77,5.12,0.15)$ \\
\hline $2+$ cells & $1.78(0.92,3.44,0.08)$ & $2.50(0.97,6.50,0.06)$ \\
\hline $3+$ cells or greater & $2.38(1.09,5.21,0.03)$ & $4.62(1.49,14.34,0.008)$ \\
\hline Anterior chamber flare $(\geq 1+$ flare $)$ & $1.79(0.84,3.83,0.13)$ & \\
\hline Prior intraocular surgery (includes cataract surgery in fellow eye) & No cases $/ /$ & \\
\hline Systemic corticosteroids at presentation & $0.86(0.08,8.68,0.90)$ & \\
\hline Topical corticosteroids at presentation & $2.50(1.46,4.27,0.001)$ & $2.12(1.37,3.27,0.001)$ \\
\hline Immunosuppressive drugs at presentation & $1.08(0.22,5.25,0.93)$ & \\
\hline \multicolumn{3}{|l|}{ ANA $=$ antinuclear antibody } \\
\hline \multicolumn{3}{|c|}{ Crude $\mathrm{RR}=$ relative risk $(95 \% \mathrm{CI}=95 \%$ Confidence interval, $\mathrm{P}$-value $)$ for univariate analyses of single exposure variable. } \\
\hline \multirow{2}{*}{\multicolumn{3}{|c|}{$\begin{array}{l}+ \text { Adjusted } \mathrm{RR}=\text { relative risk }(95 \% \mathrm{CI}=95 \% \text { Confidence interval, } \mathrm{P} \text {-value }) \text { for multivariate analyses in which all exposure variable with } \mathrm{P} \text {-values } \leq \\
0.10 \text { in the univariate analysis were included, and stepwise regression utilized that eliminated all variables with } \mathrm{P} \text {-values } \geq 0.05 \text {. }\end{array}$}} \\
\hline & & \\
\hline${ }^{+} \mathrm{P}$-value for trend in the multivariate analysis $\left.=0.005\right)$ & & \\
\hline
\end{tabular}


Table 3

Multivariate time-dependent analysis of use of topical corticosteroids and the development of cataract during follow up in patients with juvenile idiopathic arthritis-related uveitis

\begin{tabular}{lccc}
\hline Characteristic & Relative risk & 95\% Confidence interval & P-value \\
\hline Use of topical corticosteroids (<3 drops daily vs. > 3 drops daily) & 0.13 & $0.02,0.69$ & 0.02 \\
Use of other forms of corticosteroids (yes vs. no) & 1.56 & $0.13,20.0$ & 0.73 \\
Use of immunosuppressive drug therapy (yes vs. no) & 0.96 & $0.31,2.94$ & 0.94 \\
Presence of active uveitis (yes vs. no) & 6.44 & $1.19,35.0$ & 0.03 \\
\hline
\end{tabular}

